\title{
Unusually low airglow intensities in the Southern Hemisphere midlatitude mesopause region
}

\author{
Jürgen Scheer and Esteban R. Reisin \\ Instituto de Astronomía y Física del Espacio, PRONARP - CONICET, C.C. 67, Suc. 28, 1428 Buenos Aires, Argentina
}

(Received August 4, 1998; Revised January 31, 2000; Accepted February 15, 2000)

\begin{abstract}
During the first part of a new set of airglow observations in the $\mathrm{OH}(6-2)$ and the $\mathrm{O}_{2}(0-1)$ Atmospheric bands from El Leoncito, Argentina, that started in August 1997, periods of surprisingly low intensities stand out. For many nights, mean $\mathrm{O}_{2}$ intensities reach only $30 \%$, or less, of what we have seen in previous campaigns, from 1984 to 1994, at the same site, or a similar latitude. Such an anomaly is also observed, to a lesser degree, in OH intensities. An instrumental artifact in the intensity results can be ruled out by using the signal from the stellar background for a "field calibration". A relation to the strong ENSO event with its major climate impact in 1997/8, may, of course, be suspected, although a statistical inference would be weakened by the scarceness of historical precedents of a similar magnitude.
\end{abstract}

\section{Introduction}

The variability of airglow originating in the mesopause region has been the primary concern of investigations since Lord Rayleigh's green line observations starting in the early twenties (see Hernandez and Silverman, 1964). A complete interpretation of the phenomenology of airglow variations has, however, not yet been achieved (see Forsyth and Wraight, 1987, for a review of observational results). It is now believed that this is not so much due to an incomplete understanding of airglow chemistry, but because changes in airglow emissions are strongly controlled by dynamics, involving local variations in wave activity and also large-scale variations in circulation, and thus reflect a good part of the complexity of the atmospheric system, as a whole.

While the message included in airglow intensities was unclear, investigators attempted to incorporate other parameters in the analysis, that have an essential relation to dynamics and promised to be easier to interpret. This expectation has driven the development of airglow instruments that observe rotational temperatures. For this purpose, only relative (but spectrally resolved) airglow brightness measurements are needed. In consequence, airglow intensities turn into a by-product of temperature observations, overshadowed by the primary data product with its apparently more direct physical relevance to the structure and dynamics of the mesopause region.

However, theoretical and experimental advances in recent years (see, e.g., Reisin and Scheer, 1996, and references therein) have underlined the importance of combining the information from variations in both, airglow intensity and temperature, to learn more about the wave phenomena that drive them. While this has been demonstrated for the timescale of a

Copy right (C) The Society of Geomagnetism and Earth, Planetary and Space Sciences (SGEPSS); The Seismological Society of Japan; The Volcanological Society of Japan; The Geodetic Society of Japan; The Japanese Society for Planetary Sciences. night, the usefulness of taking more parameters into account is obvious, also for the slower variations, although making good use of the additional information is by no means trivial.

Our previous measurements, in Argentina, of rotational temperatures derived from the $\mathrm{OH}(6-2)$ and the $\mathrm{O}_{2}(0-1)$ Atmospheric bands, done in several campaigns between 1984 and 1994, have supplied data on two different altitude levels in the mesopause region, namely at about $87 \mathrm{~km}$ and $95 \mathrm{~km}$. The focus was on the fast variations. Although these observations did not have the objective of studying long-term airglow brightness trends, and therefore lack an absolute intensity calibration, this turns out not to be a serious limitation for studying slower intensity variations, as well. When the measurements were continued in August of 1997, after improvements in the instrument, we were surprised by the extremely weak brightness of the airglow emissions, especially of the $\mathrm{O}_{2}$ band, and the persistence of this situation, for more than one month. Even more persistent (although less pronounced) were the unusually low $\mathrm{OH}$ intensities.

Spells of low airglow intensity lasting for several hours or even several nights are not unusual and have often been observed before. On this time scale, variations corresponding to a factor of more than five are not uncommon (e.g., Scheer and Reisin, 1990, 1998).

The purpose of this paper is to report on the details of the observed anomaly and attempt an explanation. Temperatures are not discussed here, except in passing, because they will be treated in a forthcoming paper.

\section{Instrumentation and Data Quality}

The instrument used was the zenith-looking tilting filter spectrometer (described by Scheer, 1987) designed to measure fast rotational temperature variations at the $\mathrm{OH}$ and $\mathrm{O}_{2}(0-1)$ emission heights. The instrument has recently been upgraded from the obsolete Tandy microcomputer to PC control, thus opening the way to nearly automatic operation that 


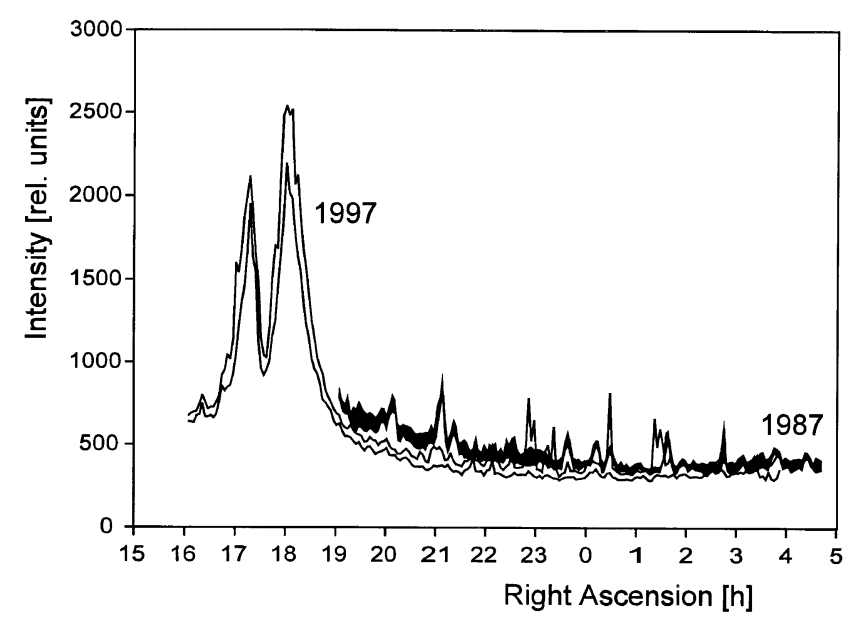

Fig. 1. Comparison of stellar background during the 1987 and 1997 campaigns, vs. right ascension. The shaded area (for 1987) and the area enclosed between two lines (for 1997) cover the two center quartiles of the data. The two principal peaks correspond to the galactic center region.

has led to a considerable increase in the amount of data acquired in the recent years.

Shortly before the observations in 1997, the instrument was calibrated with a new filter (with characteristics similar to the one previously used, by the same manufacturer). The same calibration procedures as those outlined in Scheer (1987) were used to determine the relevant spectral parameters of the complete instrument. Only the absolute intensity scale is unknown. However, we have verified by means of the signatures of the sky background that the scale factor has not changed.

By looking at the zenith from the same site, the same celestial latitude (declination) zone has always been scanned. Figure 1 shows an example of a comparison of the spectral background - corresponding essentially to starlight—-for selected moonless nights from the 1987 and 1997 campaigns (see below), with respect to celestial longitude (right ascension). The 1987 data are for September, and therefore do not include the galactic center region. We do not show the only pre-97 campaign that did so (June/July 1986), because the sky background was not included in the sampling scheme then used. Since in a scatter plot of the about ten thousand individual points the two groups of data would be difficult to distinguish, the figure only shows the areas occupied by the two central quartiles (where 1/4 of the data are excluded, on each side) of the data of such a plot (the shaded area, for 1987, and the area between the two border lines, for 1997).

One sees little offset between both data sets that could easily be ascribed to a change in instrument sensitivity: the data for 1987 and 1997 even overlap, in one part of the figure, and diverge by only about $20 \%$, in another part. Small errors in zenith alignment, comparable to the meridional extension of the field of view $\left(0.4^{\circ}\right)$, certainly contribute to the discrepancy in the shapes of both curves (at least, they led to the difference of individual star signatures, in the figure). There might also be a contribution from zodiacal light. In plots for other campaigns (especially a new one done with the data of later in 1998; not shown) such a discrepancy is not present. We can therefore conclude that the instrument sensitivity is presently quite similar to what it was, in the past, and that it has not dropped by more than, say, $20 \%$. Therefore, it is possible to directly compare our band intensity measurements from different campaigns.

In this spectral range, intensities are affected by atmospheric absorption only in the presence of thick cloud cover, but the small number of cases when this has occurred is easily detected in the background signal, and eliminated. Background corrections in the retrieval of temperatures and band intensities also leave the measurements essentially undisturbed by background effects from starlight, moonlight, andimportant for the Buenos Aires campaign in 1994-artificial city light, if the background does not become excessively high.

\section{Observations}

Campaign averages for our campaigns from El Leoncito (LEO, 31.8 ${ }^{\circ} \mathrm{S} 69.2^{\circ} \mathrm{W}$ ), Buenos Aires (BUE, 34.6 ${ }^{\circ} \mathrm{S} 58.4^{\circ} \mathrm{W}$ ), and El Arenosillo (Spain; ARE $37.1^{\circ} \mathrm{N} 6.7^{\circ} \mathrm{W}$ ) are shown in the upper and center parts of Fig. 2. Details about the campaigns, number of nights with usable data, and the approximate number of data, in each of the four observed parameters (temperature and band intensity for the $\mathrm{OH}$ and $\mathrm{O}_{2}$ emissions), are given in Table 1 (two other campaigns, in 1984 and 1988, are not included here because of the small amount of data involved). The number of nights acquired before 1997 is already quite respectable, but it represents only a small fraction of the corresponding decade. It is therefore difficult to draw conclusions with respect to what happened between campaigns; on the other hand, the good coverage of most of the individual campaign time spans leaves no doubt that each campaign can be well represented by its respective average. Note that each average may be affected by seasonal (and other) variations, as discussed in the next section. The measurements since 1997 are much more closely spaced, with a transition to quasi-continuous operation, in 1998. After our results were first presented at the DYSMER symposium in March 1998, the discussion was extended to include further data. The April to May 25 interval is defined as the last campaign, for practical reasons, although it is contiguous to the following sequence of data that extends into October of 1999 (see Table 1).

The top and center panels in Fig. 2 show the campaign averages of $\mathrm{O}_{2}$, and $\mathrm{OH}$, intensities, respectively. The average of the data from El Leoncito and Buenos Aires taken before 1997 (that is, including all our previous data from the same latitude zone) is marked by a dashed line. Although these lines are only meant as a reference, they also represent the grand average fairly well, which includes the many additional data acquired during 1998 and 1999.

For $\mathrm{O}_{2}$, a few campaign averages fall considerably above the reference line. There are several possible causes for this, such as solar activity, but these cases do not concern us here. In contrast, the first point for 1997 exhibits the lowest campaign average of all ( $56 \%$ of the reference line). The more recent data (as discussed below) indicate that during 39 nights for the same season in 1998, intensities were again relatively low (78\%), although not as low as in 1997. At mid-latitudes, an $\mathrm{O}_{2}$ band intensity deficit as pronounced and, especially, as persistent as the one in August 1997 seems to be unusual. 
Table 1. Definition of Campaigns

site / time span

LEO 1986 Jun26-Jul14

LEO 1986 Oct26-Nov10

LEO 1987 Sep14-Sep30

ARE 1990 Jan18-Mar14

LEO 1992 Oct10-Oct26

BUE 1994 Oct27-Dec07

LEO 1997 Aug05-Oct09

LEO 1997 Nov18-Jan12

LEO 1998 Apr02-May25

total:

quasi-continuous measurements

LEO 1998 May26-Dec20

LEO 1999 Jan05-Oct17

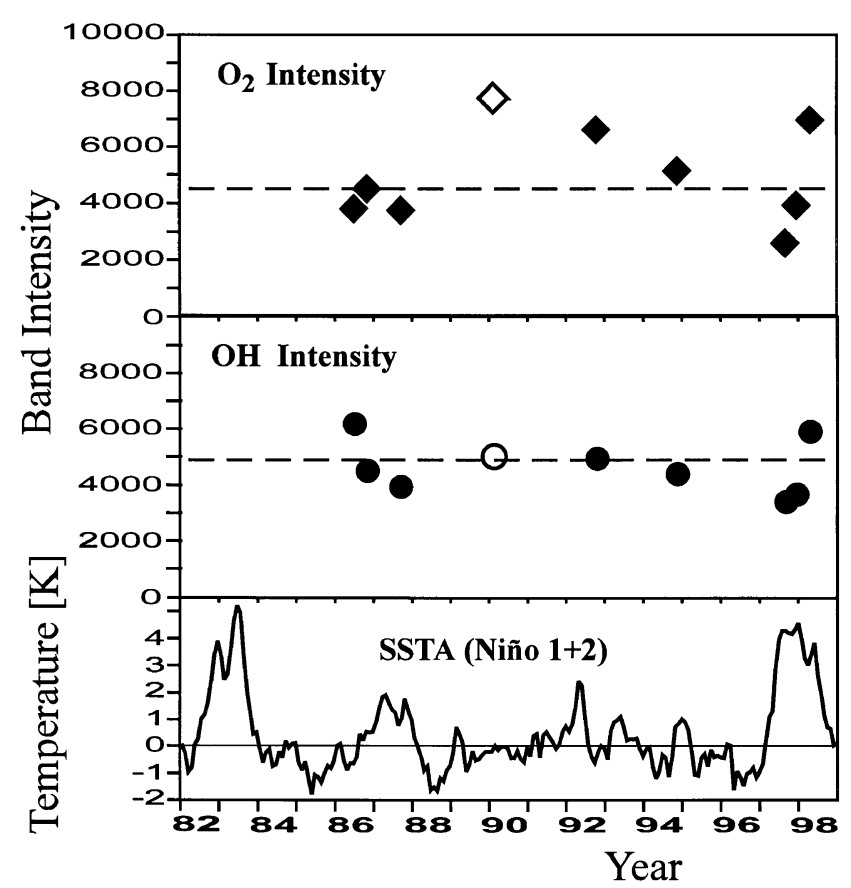

Fig. 2. Campaign averages of $\mathrm{O}_{2}$ (upper panel) and $\mathrm{OH}$ (center panel) band intensities during campaigns at El Leoncito (in 1986, 1987, 1992, and 1997/8) and Buenos Aires (in 1994; black symbols), and at El Arenosillo, Spain (in 1990; hollow symbols) obtained with the same instrument. Lower panel shows eastern equatorial Pacific Sea Surface Temperature Anomaly from NOAA/NIC's web site.

The deficit disappears at the end of the year, and the data for April/May 1998 already amply surpass the average. As we will show below, the August 1997 deficit is not just a consequence of the seasonal cycle.

For $\mathrm{OH}$ intensity, the minimum campaign averages are reached in the first and second 1997 campaigns (see Fig. 2). However, the differences are smaller than for $\mathrm{O}_{2}$, and would not appear significant, at first sight.

The distinctive characteristics of the 1997 results, in comparison with the previous campaigns and the data of the following years, come out more clearly as nocturnal averages versus time of the year. The August 1997 airglow anomaly can best be seen in the behaviour of $\mathrm{O}_{2}$ intensity, as shown in Fig. 3 (in units of the long-term average already mentioned). This, and the following figure, only show the El Leoncito data, but now including the recent quasi-continuous measurements. It should be noted that most of these data points are based on about 300 , and often more than 450 , individual observations, covering essentially all of the night; therefore, error bars would not exceed the size of the symbols in the figures. To attenuate the very strong day-to-day variability, 29-day running means (weighted by the number of nocturnal data) are also shown, whenever there are sufficient data points available. The 29-day filter length was chosen as a compromise between smoothness and loss of detail. To emphasize the 1997 anomaly, this year is represented by a separate running mean (red curve). The good consistency between the data for the other years makes it reasonable to represent them all by another single curve (magenta).

In the first part of August, the intensity is at a minimum, with night means as low as $20 \%$ of the long-term average. Except for a few isolated nights, the intensity remains low during all of August, with a monthly mean of $46 \%$ of the longterm average. There is a trend towards normal conditions, but the data start to become comparable to those of a previous campaign (1987; see Fig. 3) only at the end of September. The long-term average is not reached until the beginning of October, but intensities are normal, in November. Intensities in January drop again, but because of the following data gap, we cannot know whether this is a systematic tendency. The data return to very high intensities, in April 1998, with some nights even rivalling the maximum intensities we observed from Spain (in February 1990; Scheer and Reisin, 1998). In this month, the mean intensities even surpass the level of the following year.

The behaviour of $\mathrm{OH}$ intensities is shown in Fig. 4. Except some higher individual values at the end of November, which are not reflected in the running mean, the 1997 and January 1998 data are persistently low, at about $70 \%$ of the long-term mean, but not far below the relatively low values of the 1987 campaign. This can also be seen in the very stable 1997 running mean. In April 1998, the deficit has vanished.

\section{Discussion}

Our data show low intensities, especially during August 1997, which we find unusual. Since there is no hint of the onset of the anomalous conditions, they must already have been present at the beginning of the new data set. In fact, the HRDI instrument on the UARS satellite observed very low intensities of the (closely related) $\mathrm{O}_{2}(0-0)$ band throughout the Southern Hemisphere, in June/July, even lower than HRDI observations in August (J. Kafkalidis, private communication). Note that this is in complete contrast to the behaviour we have observed in June/July of 1986, 1998, and 1999 (Fig. 3). This means that the $\mathrm{O}_{2}$ intensity deficit must have started earlier, and its duration was at least three months.

A (semiannual) seasonal variation, with maxima at (about one month after) the equinoxes and minima in summer and winter, like the "classical" shape for $\mathrm{O}_{2}$ intensities (and the strongly correlated OI green line; e.g., Fukuyama, 1977; Garcia and Solomon, 1985; Takahashi et al., 1986, 1995) is 
certainly contributing to the low winter intensity. In Fig. 3, one can see a minimum from late July through August, a strong maximum in April, a weak one in October, and probably (not well documented) another minimum in January. These features are similar to the seasonal variation reported by Takahashi et al. (1986), for $23^{\circ} \mathrm{S}$.

To find out whether the low intensities are just a consequence of this seasonal variation, or if there is indeed an additional deficit not accountable for by a seasonal effect, we want to compare quantitatively with other midlatitude observations. This cannot be done directly because our data are not expressed in Rayleighs, but also because of the scarcity of other $\mathrm{O}_{2}$ observations close to our latitude. Therefore, we compare ratios between maxima and minima; this has the advantage of being independent of the intensity scale and allows comparison with other (correlated) emissions like the green line. Some smoothing at the order of weeks, or a month, is necessary to reduce the impact of the variability of individual nocturnal means that may vary by as much as a factor of eleven (as shown in Fig. 3).

A maximum to minimum ratio of 3.7 follows from our averages for August 1997 (0.45) and April 1998 (1.7). The data by Takahashi et al. (1986) showed a variation by a factor of two. For Adelaide, at a latitude even closer to ours, also a ratio of two can be seen in the data reported by Hecht et al. (1997), although the seasonal behaviour, then, suggested an annual rather than semiannual variation. For the green line, observations at many more sites are available. None of the 13 sites between $7^{\circ}$ and $44^{\circ} \mathrm{N}$ analyzed by Fukuyama (1977) exceeded a factor of two, except Niigata (but only slightly).

That is, our seasonal ratio significantly exceeds these other values. Since the ratio is most sensitive to a difference in the minimum, it seems a good guess to attribute the excess to the low August 1997 intensities, rather than the April 1998 maximum. This is also suggested by our more recent data, for August 1998 (an average of 0.73, that is 62\% higher than in August 1997) and April 1999 (1.46, only 14\% less than April 1998). We therefore believe that a normal seasonal variation is not sufficient to explain the low $\mathrm{O}_{2}$ intensities in winter 1997, although the anomaly may have consisted of an extraordinary seasonal amplitude.

With regard to $\mathrm{OH}$ intensities, the anomaly comes out quite clearly by comparison with data of the corresponding time spans of the next two years (see Fig. 4). The normalized averages of the 1998/9 data in these periods were close to unity, confirming that the 1997/8 level was indeed a $30 \%$ deficit. In other words, $\mathrm{OH}$ intensities were systematically lower than normal, at least in August/early September 1997 and November 1997 to January 1998. The increase in April 1998 (which was well reproduced in 1999), suggests a return to undisturbed conditions, at that time.

What are the possible causes for the unusually low intensities for both emissions? They cannot be due to solar activity effects, because both 1986/7 and 1997 correspond to low activity, close to minima of the solar cycle. While the high solar activity in the 1990 and 1992 campaigns might have been responsible for part of the enhancement of $\mathrm{O}_{2}$ intensities (and also, temperatures) at that time, there was no hint at any influence on $\mathrm{OH}$. Therefore, the modest solar activity increase in early 1998, in comparison to the 1990 maximum, is certainly insufficient to account for the observed changes in airglow conditions. Neither are there intensity differences between 1998 and 1999 that could easily be attributed to solar activity effects.

Among other types of interannual variations, the quasibiennial oscillation (QBO) might have had an impact on the conditions in 1997, but the evidence over the following two years now makes this possibility look remote.

As is well known, there has been a strong deviation from normal atmospheric conditions, at least in the lower atmosphere, namely the El Niño Southern Oscillation (ENSO) of 1997/8. A convenient proxy for the degree of the corresponding climatic perturbation is the sea surface temperature anomaly (SSTA). Its main advantage over a meteorological indicator like the Southern Oscillation Index is the lower noise level. SSTA is shown in the lower part of Fig. 2. These data (from the U.S. National Oceanic and Atmospheric Administration, NOAA, http://nic.fb4 noaa.gov/ $\mathrm{data} / \mathrm{cddb} /$ ) are based on a combination of in-situ (ship and buoy) and NOAA satellite observations (Reynolds and Smith, 1994), and a climatology derived from them (Reynolds and Smith, 1995). The curve corresponds to a monthly average over the area from $80^{\circ} \mathrm{W}$ to $90^{\circ} \mathrm{W}$ longitude and from $0^{\circ}$ to $10^{\circ} \mathrm{S}$ latitude (called "Niño $1+2$ "; this one was selected among the four options supplied in the "sstoi.indices" file for being probably more pertinent to the situation in South America). The most prominent positive excursions (also with respect of their duration) in 1982/3 and 1997/8 were by far the strongest SST anomalies since the beginning of the data set, in 1949.

As shown in Fig. 2, the strong positive trend in the recent SST anomaly started in early 1997 . The SSTA parameter first got close to its maximum excursion, in June, remaining very high for the rest of 1997. It was still far above normal conditions, even in May 1998, followed by a decline until the end of the year. That is, this ENSO event completely encompasses the interval when the unusual airglow intensities were observed.

Before the 1997 El Niño, but since the start of our airglow observations, there have been only minor SST anomalies (see Fig. 2), so that the only previous situation with a similar potential impact would have been in 1982/3. The absence of previous reports in the literature about mesopause region effects does not disprove such a possibility, given the relative sparsity of airglow observations in the Southern Hemisphere.

There are different possibilities that could explain how global circulation changes in the lower atmosphere as those occurring during a strong ENSO event might affect the mesopause region. Of course, tropospheric gravity wave generation depends on weather activity, and the background wind at low altitudes modulates the vertical propagation of atmospheric gravity waves. Breaking gravity waves in turn modify the mean flow including vertical motions in the upper atmosphere and by that, airglow emissions and the vertical temperature profile. Whether low airglow brightness is correlated with high gravity wave activity, as suggested by Garcia and Solomon (1985), is still an open question-tidal activity tends to have the opposite effect. Thus, without a theoretical guide as to what effect to expect, it is therefore presently only the temporal coincidence with the observed 


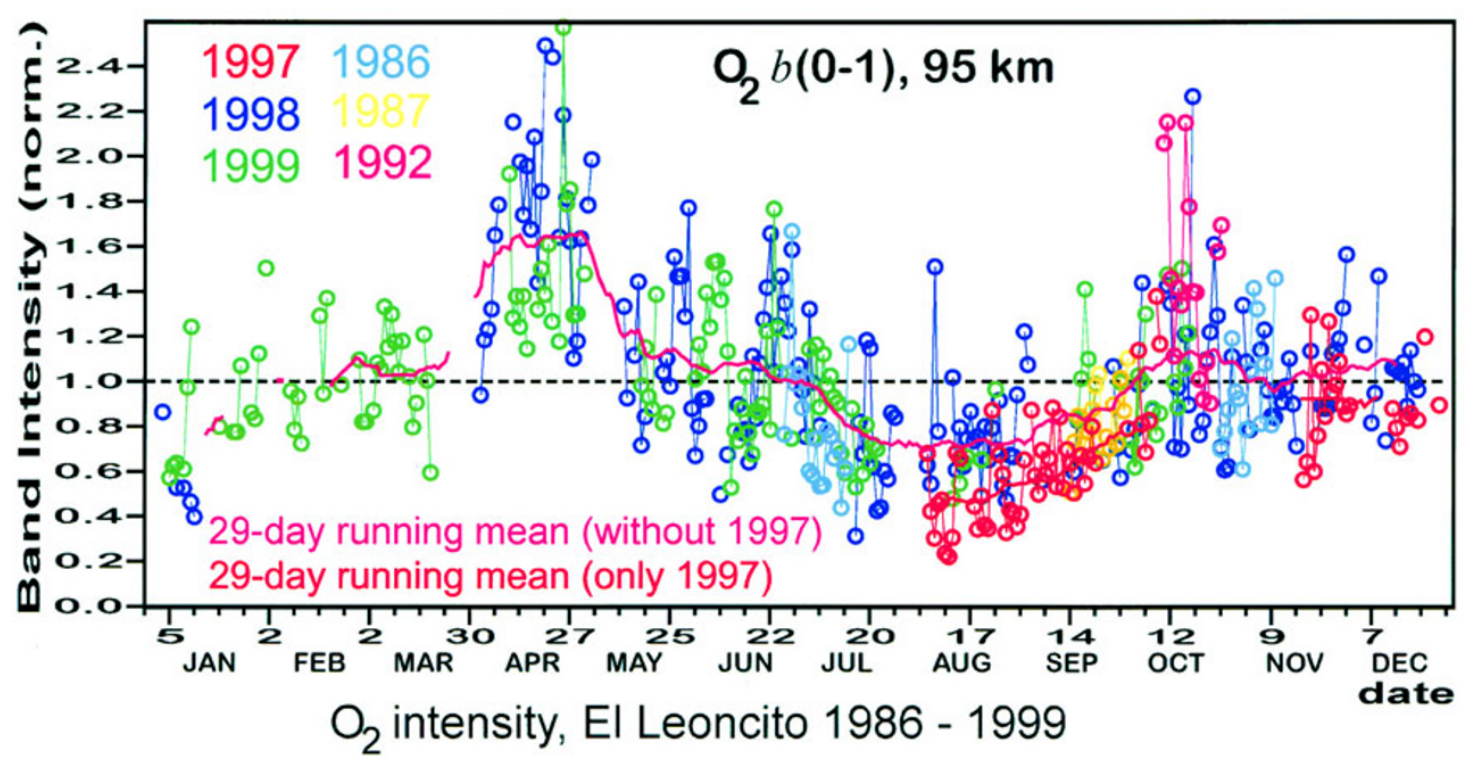

Fig. 3. Nocturnal averages of $\mathrm{O}_{2}$ band intensity, normalized by the average of the 1986, 1987, 1992, and 1994 campaigns. Points for consecutive nights are united by straight lines to guide the eye. Years are color coded. The magenta curve is the running mean for all years shown except 1997 (red curve for 1997, only).

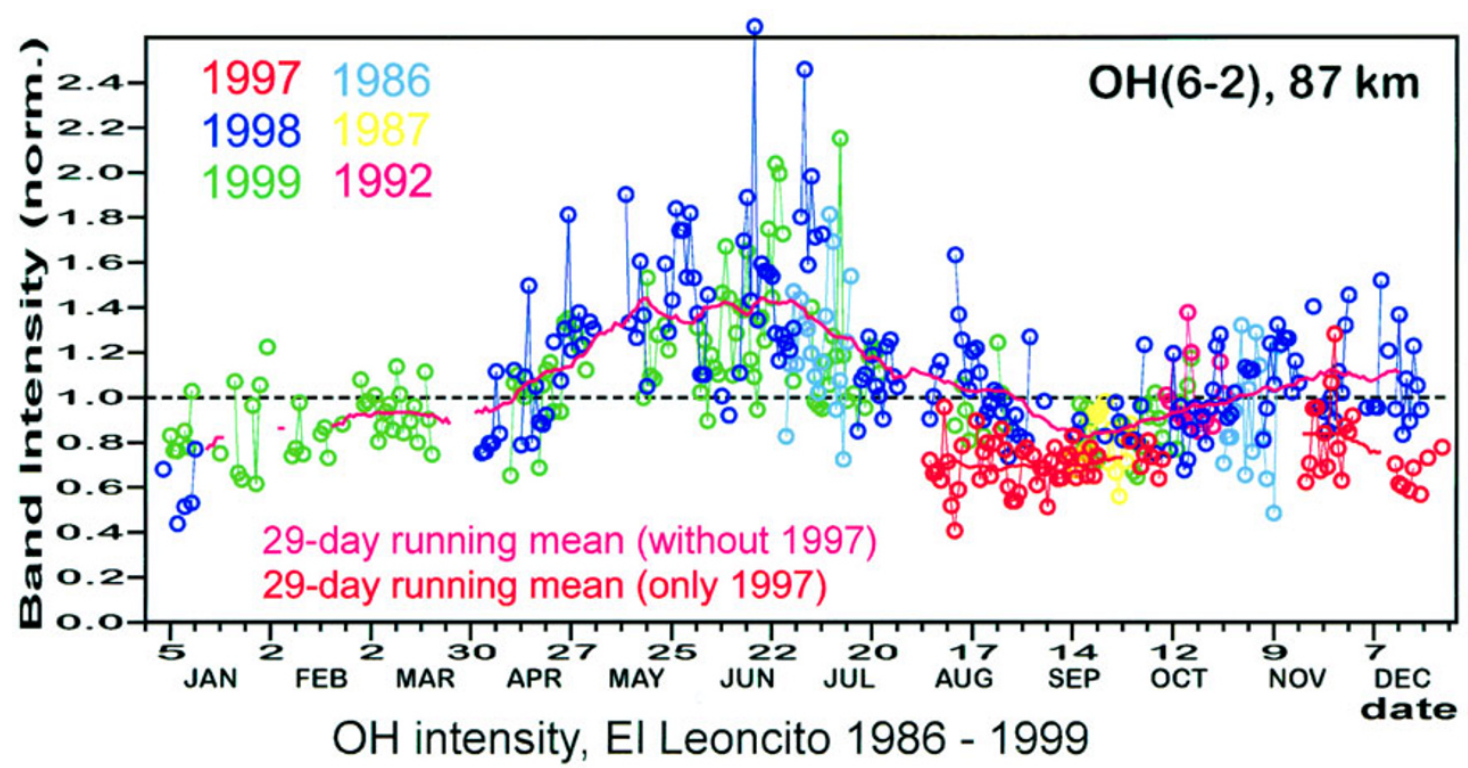

Fig. 4. Same as Fig. 3, but for $\mathrm{OH}$ band intensity.

anomaly that supports the ENSO hypothesis.

\section{Conclusions}

The comparison presented, of nocturnal and campaign averages of our observations in 1997 and early 1998, with respect to our previous and later data, has shown atypically low $\mathrm{OH}$ and $\mathrm{O}_{2}$ airglow intensities. The anomalous situation was documented in the data for several months. Seasonal variations seem incapable of explaining these effects.

The temporal coincidence with the recent extraordinarily strong El Niño climate disturbance makes it natural to expect a relationship. General circulation models (like the TIMEGCM) should be useful to check whether the propagation of tropospheric ENSO perturbations into the mesopause region can already be described with current model ingredients, or whether additional mechanisms have to be brought into play.

Given the impossibility to prove an ENSO relation, this is of course just a speculation, which further evidence might eventually confirm. Other interannual variations do not supply a more plausible explanation, although a relation to the quasi-biennial oscillation cannot be a-priori excluded.

The results from the UARS satellite, and from the second CRISTA/MAHRSI flight (in August 1997) will possibly help to shed more light on this issue, especially with respect to the geographical extension of the anomaly. There may also be more input from other observations, in the context of the Planetary Scale Mesopause Observing System. 
Acknowledgments. The authors thank the directors and staff of the Felix Aguilar / U. Cesco and CASLEO astronomical observatories at El Leoncito for technical support. Fabiana Etura Marcón contributed to get the updated instrument ready for the new observations. Guillermo Stenborg (MPAe Lindau) is also thanked for his help. We appreciate the possibility to access and use SSTA data on NOAA's internet site. This work was partially funded by CONICET and by ANPCyT grants PMP PICT 0137 and 07-00000-01818.

\section{References}

Forsyth, R. J. and P. C. Wraight, A survey of research on nightglow variability, Planet. Space Sci., 35, 1449-1461, 1987.

Fukuyama, K., Airglow variations and dynamics in the lower thermosphere and upper mesosphere-II. Seasonal and long-term variations, J. Atmos. Terr. Phys., 39, 1-14, 1977.

Garcia, R. R. and S. Solomon, The effect of breaking gravity waves on the dynamics and chemical composition of the mesosphere and lower thermosphere, J. Geophys. Res., 90, 3850-3868, 1985.

Hecht, J. H., R. L. Walterscheid, J. Woithe, L. Campbell, R. A. Vincent, and I. M. Reid, Trends of airglow imager observations near Adelaide, Australia, Geophys. Res. Lett., 24, 587-590, 1997.

Hernandez, G. J. and S. M. Silverman, A reexamination of Lord Rayleigh's data on the airglow $5577 \AA$ [OI] emission, Planet. Space Sci., 12, 97-112, 1964.
Reisin, E. R. and J. Scheer, Characteristics of atmospheric waves in the tidal period range derived from zenith observations of $\mathrm{O}_{2}(0-1)$ Atmospheric and $\mathrm{OH}(6-2)$ airglow at lower midlatitudes, J. Geophys. Res., 101, 21,223-21,232, 1996.

Reynolds, R. W. and T. M. Smith, Improved global sea surface temperature analyses, J. Climate, 7, 929-948, 1994.

Reynolds, R. W. and T. M. Smith, A high resolution global sea surface temperature climatology, J. Climate, 8, 1572-1583, 1995.

Scheer, J., Programmable tilting filter spectrometer for studying gravity waves in the upper atmosphere, Appl. Opt., 26, 3077-3082, 1987.

Scheer, J. and E. R. Reisin, Rotational temperatures for $\mathrm{OH}$ and $\mathrm{O}_{2}$ airglow bands measured simultaneously from El Leoncito (31 $\left.48^{\prime} \mathrm{S}\right)$, J. Atmos. Terr. Phys., 52, 47-57, 1990.

Scheer, J. and E. R. Reisin, Extreme intensity variations of $\mathrm{O}_{2} \mathrm{~b}$ airglow induced by tidal oscillations, Adv. Space Res., 21(6), 827-830, 1998.

Takahashi, H., Y. Sahai, and P. P. Batista, Airglow $\mathrm{O}_{2}\left({ }^{1} \Sigma\right)$ atmospheric band at $8645 \AA$ and the rotational temperature observed at $23^{\circ} \mathrm{S}$, Planet. Space Sci., 34, 301-306, 1986.

Takahashi, H., B. R. Clemesha, and P. P. Batista, Predominant semi-annual oscillation of the upper mesospheric airglow intensities and temperatures in the equatorial region, J. Atmos. Terr. Phys., 57, 407-414, 1995.

J. Scheer (e-mail: jurgen@caerce.edu.ar), and E. R. Reisin (e-mail: ereisin@iafe.uba.ar) 